\title{
Effect of High-dose Ascorbic Acid on Prostate Cancer Cells and Angiogenesis
}

\author{
(1) Naşide Mangır' ${ }^{1,2}$, (0 Sheila MacNeil ${ }^{1}$ \\ 'Sheffield University Kroto Research Institute, Department of Materials Science Engineering, Sheffield, United Kingdom \\ 2 Royal Hallamshire Hospital, Clinic of Section of Functional and Reconstructive Urology, Sheffield, United Kingdom
}

\begin{abstract}
Objective: Ascorbic acid (AA), at physiological concentrations, is a well known anti-oxidant but, at higher concentrations, it can be selectively toxic to cancer cells. Controversial data have shown that administration of intravenous high-dose AA can improve therapeutic outcomes in cancer patients. These effects are thought to occur mainly due to direct cytotoxicity mediated by generation of reactive oxygen species. The anti-tumour effects of AA can also be due to inhibition of angiogenesis, however, this has been poorly studied. This study aimed to investigate the effect of AA on prostate cancer cells and angiogenesis.

Materials and Methods: Hormone resistant and naïve human prostate cancer cell lines (PC-3 and LnCaP, respectively) were treated with different concentrations of AA. Cell viability was determined by the resazurin assay. Polymer scaffolds that releases lower $(0.01 \mathrm{~g} / \mathrm{g}$ polymer) and higher ( $0.1 \mathrm{~g} / \mathrm{g}$ polymer) concentrations of AA were used to evaluate the effects of AA on angiogenesis. Angiogenesis was investigated by an in vivo chick chorioallantoic membrane assay.

Results: AA was toxic to PC-3 and LnCaP prostate cancer cell lines in a dose-dependent manner. Confluent cultures of LnCaP was more sensitive to high-dose AA, while confluent cultures of PC-3 were resistant. There was a biphasic in vivo angiogenic response to AA, that is, while high-dose of $A A$ inhibited angiogenesis, low dose AA stimulated angiogenesis. In addition, a stable form of AA (ascorbate-2 phosphate) was not angiostatic at high concentrations, suggesting that angiostatic actions of high-dose AA may be due to its direct toxicity on endothelial cells.

Conclusion: We demonstrated that AA was toxic to prostate cancer cell lines, and inhibits angiogenesis at high doses. Further in vivo studies are required to validate these finding before any clinical inference can be made.
\end{abstract}

Keywords: Angiogenesis, ascorbic acid, prostate cancer

\section{Introduction}

Prostate cancer (PCa) is the most common type of cancer that affects male adults. In Turkey, PCa is the most prevalent cancer in men after lung cancer, with 17,332 new cases recorded per year (1). Deaths due to PCa accounts for $1.5 \%$ of all deaths in men (2). At the time of diagnosis, $80 \%$ of men diagnosed with PCa have organ confined disease which is can be treated by surgery and/or radiotherapy. However, some patients may subsequently develop systemic disease with distal organ involvement. Metastatic PCa responds sensitively to androgen deprivation therapy initially, but resistance to the hormone develops few years later, resulting in a castration resistant PCa which requires systemic chemotherapy. Docetaxel based chemotherapy improves overall survival in patients with metastatic castration resistant $\mathrm{PCa}$, and is therefore the standard first-line treatment for these patients (3). However, there are limited options for patients whose PCa develops into disease progression after the docetaxel treatment. In this regard, better treatments modalities that can increase patient survival or quality of life are currently being investigated.

Ascorbic acid (AA), either alone or as a supplementary agent, has been put forward as a treatment option for patients with advanced PCa. AA is one of the essential vitamins in humans, and it is best known for its role in regulating the biosynthesis of collagen. Anti-tumour effects of high-dose AA was first reported in the 1970s (4). Unfortunately, subsequent clinical trials failed to demonstrate any therapeutic benefit of AA in patients with advanced cancers $(5,6)$. These controversial findings are thought to be due to the differences in pharmacokinetics of AA when administered orally or intravenously (7). Recent evidence from clinical studies suggests a potential synergistic effect of high-dose intravenous $A A$ and other chemotherapeutic agents (8). For treatment of PCa, AA was demonstrated to effectively

Cite this article as: Mangır N, MacNeil S. Effect of High-dose Ascorbic Acid on Prostate Cancer Cells and Angiogenesis. Bull Urooncol 2020;19(3):107-111

Address for Correspondence: Naşide Mangır, Sheffield University Kroto Research Institute, Department of Materials Science Engineering and Royal Hallamshire Hospital, Clinic of Section of Functional and Reconstructive Urology, Sheffield, United Kingdom E-mail: nasidemangir@yahoo.com ORCID-ID: orcid.org/0000-0002-3062-6480 Received: 26.05.2020 Accepted: 18.06.2020 
inhibit cell division and DNA synthesis in vitro $(9,10)$, and also decrease tumour growth in animal models (11). Available clinical data revealed that high-dose intravenous $A A$ can safely be administered in PCa patients (7), with some clinical benefits (12). However, recent evidence does not support the usage of high-dose intravenous AA in patients with PCa (13). Therefore, the role of high-dose AA in treatment of patients with advanced PCa is still controversial.

Another contributing factor on this controversy has to do with the less investigated anti-tumour aspect of $A A$, that is, its effect on angiogenesis. All tumours require a blood supply to grow in vivo. Hypothetically, the therapeutic effects of AA treatment in cancer patients could be mediated via modification of angiogenesis in the tumour. Moreover, AA plays a key role in the synthesis and post-translational modification of collagen III and IV, which are the main structural proteins that form the wall of blood vessels and the basement membrane. AA has also been previously suggested as a factor involved in angiogenesis $(14,15)$. The effects of AA on angiogenesis is not well investigated as studies have only reported the angiostatic (16) and proangiogenic effects (17).

The aimed to investigate the anti-tumour effects of $A A$. We have initially examined the effect of high-dose AA on two PCa cell lines, and then investigated the angiogenic effect of different doses of AA in vivo.

\section{Materials and Methods}

\section{Culturing of PCa Cell Lines}

This is an experimental study using human cell lines. Two human PCa cell lines were used to investigate the toxic effects of AA. The PC-3 cell line represented the hormone resistant PCa, while $\mathrm{LnCaP}$ represented the hormone naïve PCa. We obtained this cell lines as a generous donation from doctor Adam Glen [who initially purchased them from the American Type Culture Collection (Manassas, VA)]. Both cell lines were cultured in T75 flasks and maintained in RPMI-1640 medium supplemented with fetal calf serum (10\% v/v), L-glutamine $(2 \mu \mathrm{M})$, Penicillin $(100 \mathrm{U} / \mathrm{mL})$, Streptomycin $(100 \mathrm{ug} / \mathrm{mL})$ and Fungizone $(0.625$ $\mathrm{ug} / \mathrm{mL})$, all from Sigma-Aldrich.

Cultures of PC-3 and LnCaP were plated at a concentration of 50,000 cells per well in a 12 -well plate. Proliferating cultures were treated with AA at 24 hours, while confluent cultures were treated with $A A$ at 72 hours. Solutions of $A A$ were prepared in distilled water with concentrations of $0,50,100,250,500$ and $1,000 \mu \mathrm{M}$, and were freshly used. At the start of the experiments, the culture media were removed from the plates and the wells were washed three times with phosphate buffered saline (PBS). Freshly prepared solutions of AA were added into each plate and left in an incubator for 2, 4 and 6 hours. All experiments were repeated thrice.

\section{Assessment of Toxicity}

AA toxicity was assessed using a resazurin assay which essentially measures cell metabolic activity. In briefly, at 2, 4 and 6 hours, all media were removed from the culture plates and PBS was used to wash the wells. Then, $5 \mathrm{~mL}$ of resazurin solution $(125 \mu \mathrm{l} / \mathrm{mL}$ resazurin in PBS) was added into each well and incubated for 45 minutes at $37{ }^{\circ} \mathrm{C}$. Absorbance was then measured at $570 \mathrm{~nm}$ with a colorimetric plate reader (Bio-TEK, NorthStar Scientific Ltd, Leeds, UK). Culture wells without cells were used as blanks.

\section{Achieving a Continuous Release of High-dose AA from Polymers}

Studying the effect of $A A$ on angiogenesis requires a continuous drug-release system that can effectively deliver high-dose AA into the cells. This was achieved by using a polymer as the drug carrier as previously described (18). In briefly, an electrospun polylactic acid (PLA) was used as the drug carrying polymer for AA. A technique of emulsion electrospinning was employed to obtain a stable solution of $\mathrm{AA}$ and the polymer. Drug releasing PLA materials were prepared in two different concentrations including 0.01 (low dose) and 0.1 (high-dose) grams of the drug per gram of PLA polymer. A salt formulation of AA [L-ascorbic acid 2-phosphate (A2P)] was used as a control to test the difference between the active form of $A A(L-A A)$ and the salt form. PLA scaffolds releasing hydrocortisone were also constructed to serve as a negative control. This is hydrocortisone is a known angiostatic agent with an appreciable solubility in lipid, making it an excellent candidate for the negative control. All reagents and chemicals were obtained from Sigma-Aldrich, United Kingdom.

\section{Studying Angiogenesis}

The effect of $\mathrm{AA}$ on angiogenesis was studied ex-ovo using a chick chorioallantoic membrane (CAM) assay described previously (19). All embryo cultures were covered under a home office licence. Briefly, fertilised chicken eggs (gallus gallus domesticus) were cultured in a rotating egg incubator till day 3 of the embryonic development. The embryos were then transferred into petri dishes in order to continue the embryo culture ex-ovo in a humidified cell culture incubator at $38^{\circ} \mathrm{C}$. The AA releasing scaffolds were cut into $7 \mathrm{~mm}$ diameter circles with a laser cutter device [Epilog Laser Cutter (Clevedon, UK)]. Sterilisation of the drug releasing polymers were performed via exposure to ultraviolet light for 30 minute. At day 7 of the embryonic development, the test samples were placed on the CAM surface and cultured for 4 days.

At day 11 of embryonic development, the area of the CAM containing the test samples were photographed with a digital microscope. Afterwards, the cultures were terminated by sacrificing the chick embryos.

Digital photographs of the area of interest were taken at the end of the experiment. A total of 6-8 photographs were evaluated for each group. The digital photographs were converted to grayscale, and the contrast was enhanced using Imagel software and NeuronJ, as described previously (20). All discernible blood vessels in the photographs were marked using the NeuronJ tracing tool. The total number of blood vessels, total length of blood vessels and mean length of blood vessels were calculated using the processed images.

\section{Statistical Analysis}

Statistical analysis was performed with SPSS version 17.0 (SPSS Inc., Chicago, III., USA). Differences between groups were tested 
for statistical significance by Mann-Whitney $U$ test. $P$ values $<0.05$ was considered significant.

\section{Results}

\section{Effect of AA on PC3 Cells}

Supplementation of AA on proliferating cultures of PC3 cells at concentrations of $0,50,100,250$ and $1,000 \mu \mathrm{M}$ resulted in a dose dependent reduction in cell viability as measured by the metabolic activity of the cells. AA concentrations $>250 \mu \mathrm{M}$ were the most effective to reduce PC3 cell viability. There were no significant differences between measurements taken at 2, 4 and 6 hours. On the other hand, the same concentrations of AA had no significant effect on cell viability of the confluent cultures of PC3 cells (Figure 1).

\section{Effect of AA on LnCaP Cells}

Supplementation of $\mathrm{AA}$ on both proliferating and confluent cultures of LnCaP cells at concentrations of $0,50,100,250$ and $1,000 \mu \mathrm{M}$ resulted in a dose dependent reduction in cell viability as measured by the metabolic activity of the cells. AA Concentrations $>250 \mu \mathrm{M}$ were the most effective to reduce LnCaP cell viability (Figure 2).

\section{Effect of AA on Angiogenesis}

The effect of AA on angiogenesis was investigated using a PLA electrospun polymer system that ensures continuous release of AA at low (0.01 g/g PLA) and high ( $0.1 \mathrm{~g} / \mathrm{g}$ PLA) concentration. It is important to continuously release the AA on the experimental
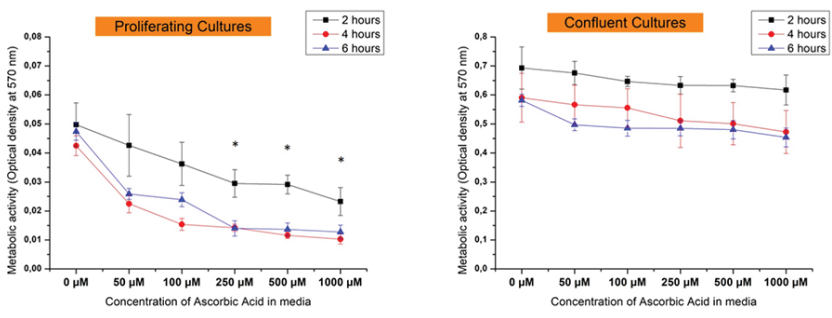

Figure 1. Assessment of different concentrations $(0-1000 \mu \mathrm{M})$ of ascorbic acid (AA) on proliferating and confluent cultures of PC3 cells at different time points. There was a dose-dependent decrease in the metabolic activity of PC3 proliferating cells, particularly above concentrations of $250 \mu \mathrm{M}$. The confluent cultures of PC3 cells were resistant to the effects of high-dose AA in the cell culture medium ( ${ }^{*} \mathrm{p}<0.05$ as compared to a concentration of $0 \mu \mathrm{M}$ )
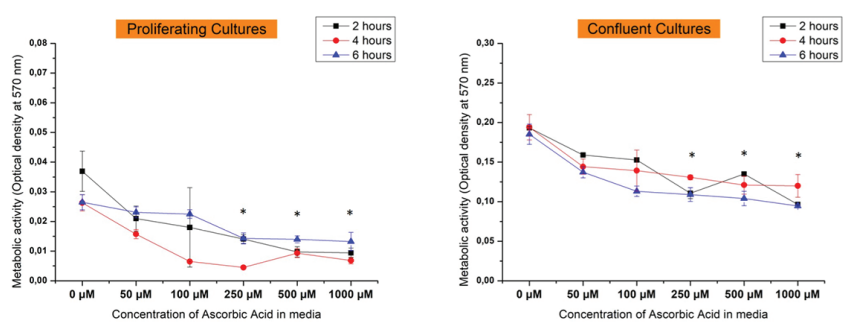

Figure 2. Assessment of different concentrations $(0-1000 \mu \mathrm{M})$ of ascorbic acid (AA) on proliferating and confluent cultures of LnCaP cells at different time points. There was a dose-dependent decrease in the metabolic activity of both proliferating and confluent cultures of LnCaP cells, starting from a dosage of 100 $\mu \mathrm{M}\left({ }^{*} \mathrm{p}<0.05\right.$ as compared to a concentration of $\left.0 \mu \mathrm{M}\right)$ medium since AA is immediately oxidised after being applied. In these experiments plain PLA scaffolds were used as controls and hydrocortisone releasing PLA scaffolds were used as negative control to show the reaction with a known angiostatic agent (Figure 3).

High-dose of AA showed an angiostatic effect, with a significant decrease in total number of blood vessels $(35.44 \pm 2.56$ vs $85 \pm 5.66, p<0.005)$ and total length of blood vessels $(11.14 \pm 0.61$ $\mathrm{cm}$ vs $19.59 \pm 0.79 \mathrm{~cm}, \mathrm{p}<0.005)$ when compared to control. AA caused the decrease in the total number of blood vessels and total length of blood vessels when compared to the negative control (hydrocortisone releasing scaffolds). However, these reductive effects were not statistically significant $(35.44 \pm 2.56$ vs $43 \pm 3.80$ and $11.14 \pm 0.61$ vs $13.67 \pm 0.91$, respectively).

In contrast to high-dose $\mathrm{AA}$, low dose $\mathrm{AA}$ exhibited a proangiogenic, causing a significant increase in total number blood vessels $(35.44 \pm 2.56$ vs $85 \pm 5.66, \mathrm{p}<0.005)$ and total length of blood vessels $(11.14 \pm 0.61 \mathrm{~cm}$ vs $19.59 \pm 0.79 \mathrm{~cm}$, $\mathrm{p}<0.005)$ when compared to control. In addition, high and low dose of L-A2P caused an similar effect to that of low dose AA, yielding a total number of blood vessels of $100.57 \pm 6.28$ and $101 \pm 4.95$ at high and low dose of A2P, respectively and a total length of blood vessels of $22.43 \pm 0.77 \mathrm{~cm}$ and $23.8 \pm 0.77 \mathrm{~cm}$ at high and low dose of $\mathrm{A} 2 \mathrm{P}$, respectively.

The mean length of blood vessels was increased in the angiostatic samples and this can be due to the lack of newly forming blood vessels which consequently leaves a scattered larger calibre of blood vessels. These scattered larger calibre of blood vessels subsequently increased the mean vessel length. Low concentrations of $\mathrm{AA}$ caused a significant increase in the total number of blood vessels.

\section{Discussion}

This study confirmed the toxic effects of high-dose AA on PCa cell lines. More importantly, this is the first study to show a

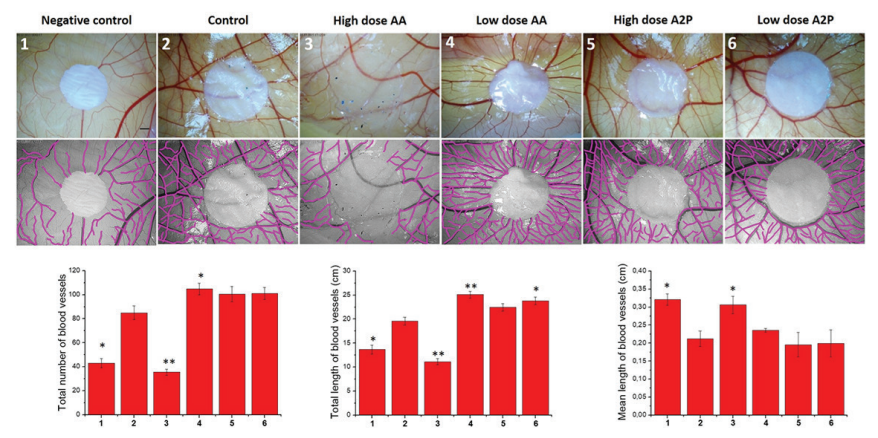

Figure 3. Demonstration of the angiogenic response to high and low doses of ascorbic acid (AA) when released from electrospun polylactic acid (PLA) scaffolds. Hydrocortisone releasing PLA scaffolds was used as negative control, whereas plain PLA scaffolds were used as controls. Additionally, the same concentrations of a more stable form of AA, L-ascorbic acid 2-phosphate (A2P) attached to the releasing scaffold was taken as another control group to check if the AA effect could be due to the acidity of the compound. High-dose AA inhibited angiogenesis, whereas low dose AA stimulated it. Both high and low doses of $\mathrm{A} 2 \mathrm{P}$ stimulated angiogenesis, suggesting that stable forms of AA are not angiostatic (error bar represents $200 \mu \mathrm{m}$ for all images) 
biphasic in vivo angiogenic response to $\mathrm{AA}$, where high doses inhibited new blood vessel formation and low doses stimulated it. This explains the contradictory reports on the angiogenic potential of AA in literature.

AA has been demonstrated to have different effects on cancer cell viability when used in high and low doses, thus suggesting a biphasic response. High doses of AA (1-10 mM) has been shown to reduce cell viability and mobility in PCa cells via production of excessive intracellular reactive oxygen species, triggering necrotic cell death (11). In contrast, low doses of AA are known to protect cells against oxidative stress. In the present study, the toxic effects of AA was seen at concentrations above $250 \mathrm{mM}$.

The mechanisms underlying AA toxicity is relatively welldefined. Although we have not studied the mechanisms behind the reduction of cell viability in PC-3 and LnCaP cell lines, the present study contributes to knowledge by demonstrating that confluent cell cultures of PC-3 cells were resistant to AA toxicity, whereas both proliferating and confluent cultures of LnCaP cell lines were equally sensitive to AA toxicity. Previous studies reported differences in response to treatment with high-dose AA between hormone treatment sensitive and resistant PCa cells (21). This implies that different cell lines can differ in terms of responsiveness to AA toxicity. This may also differ depending on the stage of the cancer cell cycle. Therefore, our findings confirm the toxicity of high-dose AA on PCa cell lines. Also, we suggest that this response can be dependent on the cell type and the stage of the cell culture.

\section{Study Limitations}

Several studies have also reported a reduction in tumour cell mass with systemic administration of AA in animal models (22). Angiogenesis is essential for tumour growth in vivo, and another mechanism for anti-tumour effects of high-dose AA could be through inhibition of angiogenesis. This has not been studied in literature, presumably because of the challenges in achieving a sustained release of AA in vivo. The present study addressed this challenge by constructing an AA releasing polymer system that ensured constant release of AA on the surface of the blood vessels, resulting in better visualisation of the effect. Taken altogether, our findings explained why AA is regarded as an angiostatic in literature (16) whereas others reported a proangiogenic effect (17). We further suggest that the contradictory results on the angiogenic potential of AA was due to difference in experimental design and dose regimens. Additionally, we provided a direct comparison of two different forms of AA (AA and A2P) at two different concentrations. To the best of our knowledge, this is the first direct in vivo comparison.

In regard to the underlying mechanisms of angiostatic effects of $A A$, we can argue that the inhibitory effect of high-dose AA on new blood vessel formation is a directly toxic to the rapidly proliferating endothelial cells of the CAM by increasing the reactive oxygen species. This argument is supported by the lack of anti-angiogenic effect of the same dose of A2P, which is a stable form of AA that does not rapidly trigger the accumulation of free radicals.

Another limitation of the available studies on the angiogenic potential of $\mathrm{AA}$ is the use of conventional $2 \mathrm{D}$ in vitro methodologies. Studying angiogenesis is complex and thus requires specialised in vivo methodologies to allow observations of interactions between endothelial and non-endothelial cells, as well as with many enzymes, growth factors and adhesion molecules that play a role in the formation of new blood vessels. This is only possible using in vivo methodologies, however, such methodology requires specialised equipment and expertise, and are often expensive (eg dorsal skin fold assay). In the current study, we made use of a modified CAM assay to study angiogenesis in a reproducible and relatively easy way (19). Our findings were obtained with a robust experimental methodology.

\section{Conclusion}

Although this study provides strong pre-clinical data on the effects of $\mathrm{AA}$ on PCa cells and on angiogenesis, it has several limitations. Firstly, the preliminary results lack confirmatory studies with larger animal models. Therefore, further studies on appropriate animal models are needed before any conclusion is reached. Secondly, the results do not imply any clinical applications at this stage. Thirdly, we have not undertaken any molecular investigation to help elucidate the mechanisms underlying the effects of AA. We believe that the methodology described in this study would trigger further experiments that will illuminate the underlying molecular mechanisms.

\section{Acknowledgements}

Publication: The results of the study were not published in full or in part in form of abstracts.

Contribution: There is not any contributors who may not be listed as authors.

Conflict of Interest: No conflict of interest was declared by the authors.

Financial Disclosure: The authors declared that this study received no financial support.

\section{Ethics}

Ethics Committee Approval: This is an experimental study using human cell lines.

Informed Consent: All embryo cultures were covered under a home office licence.

Peer-review: Externally and internally peer-reviewed.

\section{Authorship Contributions}

Concept: N.M., Design: N.M., Data Collection or Processing: N.M., Analysis or Interpretation: N.M., Literature Search: N.M., Writing: N.M., S.M.

\section{References}

1. WHO, "GLOBOCAN 2018," 2018.

2. "Türkiye İstatistik Kurumu." [Online]. Available: www.tuik.gov.tr/ PrelstatistikTablo.do?istab_id=1415 [Accessed: 17-May-2020].

3. Tannock IF, de Wit R, Berry WR, et al. Docetaxel plus prednisone or mitoxantrone plus prednisone for advanced prostate cancer. $\mathrm{N}$ Engl J Med 2004;351:1502-1512. 
4. Cameron E, Campbell A. The orthomolecular treatment of cancer. II. clinical trial of high-dose ascorbic acid supplements in advanced human cancer. Chem Biol Interact 1974;9:285-315.

5. Creagan ET, Moertel CG, O'Fallon JR, et al. failure of high-dose vitamin c (ascorbic acid) therapy to benefit patients with advanced cancer. N Engl J Med 1979;301:687-690.

6. Hoffer LJ, Levine M, Assouline S, et al. Phase I clinical trial of i.v. ascorbic acid in advanced malignancy. Ann Oncol 2008;19:1969-1974.

7. Nauman G, Gray JC, Parkinson R, et al. systematic review of intravenous ascorbate in cancer clinical trials. Antioxidants 2018;7:89.

8. Monti DA, Mitchell E, Bazzan AJ, et al. Phase I evaluation of intravenous ascorbic acid in combination with gemcitabine and erlotinib in patients with metastatic pancreatic cancer. PLoS One 2012;7:29794.

9. Mojic M, Pristov JB, Maksimovic-Ivanic D, et al. Extracellular iron diminishes anticancer effects of vitamin C: An in vitro study. Sci Rep 2015;4:5955.

10. Maramag C, Menon M, Balaji KC, et al. Effect of vitamin C on prostate cancer cells in vitro: Effect on cell number, viability, and DNA synthesis. Prostate 1997;32:188-195.

11. Ryszawy D, Pudelek M, Catapano J, et al. High doses of sodium ascorbate interfere with the expansion of glioblastoma multiforme cells in vitro and in vivo. Life Sci 2019;232:116657.

12. Mikirova N, Casciari J, Rogers A, Taylor P. Effect of high-dose intravenous vitamin $C$ on inflammation in cancer patients. J Transl Med 2012;10:189.

13. Nielsen TK, Højgaard M, Andersen JT, et al. Weekly ascorbic acid infusion in castration-resistant prostate cancer patients: a single-arm phase II trial. Transl Androl Urol 2017;6:517-528.
14. Ivanov VO, Ivanova SV, Niedzwiecki A. Ascorbate Affects Proliferation of Guinea-pig Vascular Smooth Muscle Cells by Direct and Extracellular Matrix-mediated Effects. J Mol Cell Cardiol 1997;29:3293-3303.

15. Utoguchi N, Ikeda K, Saeki K, et al. Ascorbic acid stimulates barrier function of cultured endothelial cell monolayer. J Cell Physiol 1995; 163:393-399.

16. Ashino $H$, Shimamura $M$, Nakajima $H$, et al. Novel Function of Ascorbic Acid as an Angiostatic Factor. Angiogenesis 2003;6:259-269.

17. Stumpf $U$, Michaelis $M$, Klassert $D$, et al. Selection of proangiogenic ascorbate derivatives and their exploitation in a novel drug-releasing system for wound healing. Wound Repair Regen 2011;19:597-607.

18. Mangır N, Bullock AJ, Roman S, et al. Production of ascorbic acid releasing biomaterials for pelvic floor repair. Acta Biomater 2016;29:188-197.

19. Mangir N, Dikici S, Claeyssens F, Macneil S. Using ex-ovo chick chorioallantoic membrane (cam) assay to evaluate the biocompatibility and angiogenic response to biomaterials. ACS Biomater Sci Eng 2019;5:3190-3200.

20. Eke G, Mangir M, Hasirci N, et al. Development of a UV crosslinked biodegradable hydrogel containing adipose derived stem cells to promote vascularization for skin wounds and tissue engineering. Biomaterials 2017;129:188-198.

21. Chen P, Yu J, Chalmers B, et al. Pharmacological ascorbate induces cytotoxicity in prostate cancer cells through Atp depletion and induction of autophagy. Anticancer Drug 2012;23:437-444.

22. Pollard HB, Levine MA, Eidelman O, Pollard M. Pharmacological ascorbic acid suppresses syngeneic tumor growth and metastases in hormone-refractory prostate cancer. In Vivo 2010;24:249-255. 